\title{
Simple Adaptive Control without Passivity Assumptions and Experiments on Satellite Attitude Control DEMETER Benchmark*
}

\author{
D. Peaucelle $\mathrm{t}^{\dagger \dagger \dagger}$, A. Drouot ${ }^{\dagger, \ddagger}$, \\ C. Pittet ${ }^{\ddagger}$, J. Mignot ${ }^{\ddagger}$ \\ † CNRS; LAAS; 7, avenue du Colonel Roche, \\ F-31077 Toulouse, France \\ †† Université de Toulouse; UPS, INSA, INP, ISAE; LAAS ; \\ F-31077 Toulouse, France \\ $\ddagger$ CNES; DCT/SB/PS; \\ BPI 1712, 18 avenue E. Belin, 31401 Toulouse, France
}

October 13, 2010

\begin{abstract}
The paper gives a simple LMI procedure to design simple adaptive control laws for 'almost stable' systems. It relaxes existing 'almost passive' assumptions making simple adaptive control possible for all systems stabilizable by LTI controllers. The expected advantage of the adaptive control compared to the initial LTI control is to improve performances and robustness. The proposed results apply to multi-input multi-output systems and rely on LMI optimization. The theory is illustrated on a satellite attitude control DEMETER benchmark.
\end{abstract}

\section{Introduction}

Adaptive control is a control strategy proposing to make online modifications of the control law to better reject inevitable uncertainties and perturbations. One such adaptive scheme ([IS96, ÅW89]) relies on estimating online these uncertainties and perturbations and with that knowledge to tune the control law. Another adaptation scheme ([Fra74, KBS94]) proposes to directly tune the control gains with the sole output measurements. Often called direct adaptive control it has the advantage of simplicity compared to estimation/gain-scheduling and other more complex schemes. On the other hand it has the disadvantage to require strong assumptions on the system characteristics in order to prove closed-loop stability. One such direct adaptive control scheme is the passification-based strategy (also called simple adaptive control in [KBS94]). The results presented in this paper enter this framework.

The passivity-based adaptive control framework was first restricted to square systems having same number of inputs and outputs ([Fra74]). Moreover, the systems were assumed to have open-loop hyper-minimum phase properties or equivalently in [KBS94] to be 'almost strictly passive' (i.e. being closed-loop passive with some static output feedback gain). The conditions have been mitigated in [BTH06] and relaxed in [Fra03, Fra08] to non square systems at the expense of finding a linear combination of outputs $G y$ that makes the new weighted system 'almost strictly passive'. Further extension is proposed in [PF08] by the introduction of a parallel feedforward gain (also known as a shunt, see [KBS94]). These extensions conclude that a necessary condition for passivitybased adaptive control is to have a static output feedback $u=F_{0} y$ that renders the closed-loop strictly passive

\footnotetext{
* This work was supported in part by the French Space Agency CNES.
} 
with respect to some output $G y+D u$. Unfortunately, finding these $F_{0}, G, D$ matrices appeared in that paper as a hard problem and does not even guarantee the validity of the adaptive control (the $F_{0}, G, D$ matrices have to satisfy additional LMI conditions). The goal of the present paper is to prove that as soon as a system is 'almost stabilizable' (i.e. closed-loop stable for some static output feedback gain $F_{0}$ ) then a direct adaptive control can be built.

The direct adaptive scheme we propose involves as usual in this framework a feedback control $u(t)=K(t) y(t)$ where the time-varying gain $K(t)$ is adapted according to a differential equation. This differential equation includes classically a gradient type term $-G y y^{T}$ that drives the feedback gain to stabilizing values according to closed-loop passivity properties. It also includes a second corrective term $-\phi\left(K-F_{0}\right)$ intended to keep the gain in reasonable values in practice. As it was done in [PF08] the $\phi$ function is not just a modification term assumed not to modify stability properties proved when it is put to zero, it does intervene explicitly in the closed-loop stability proof and is related to the feedthrough gain $D$.

Results for designing the adaptive law parameters are given in terms of LMIs. This not only allows to take advantage of convex optimization tools such as SeDuMi by [Stu99] and the easy to code with YALMIP interface of [Löf04], but it also paves the way for conditions guaranteeing robustness. Inspired by first results of [PFA08, BYYS07] extensions of this paper contributions to robustness issues are given in [PAMF11].

But this manuscript rather concentrates on sketching properties of the adaptive scheme on a satellite attitude control example. The full control problem with real data provided by CNES is exposed and an illustrative adaptive control law design is performed.

On-orbit life of a satellite is decomposed into phases allowing the convergence from the separation state to the mission state, orbit control, and safe state in case of fault. For each phase or 'mode', a specific set of attitude pointing requirements is to be met by using a specific set of actuators and sensors. Inside a mode, several attitude control and estimation algorithms can be designed for example to meet increasing performance levels requirements. For the past thirty years, the number of AOCS (Attitude ans Orbit Control System) modes has been reduced and now generic AOCS architecture consists typically on two or three modes (one safehold mode, one mission mode and one orbit control mode). In the same time the software complexity has increased to meet ever more demanding requirements. Thus the switching between AOCS modes and software needs more and more robustness to be able to tackle larger kinematics conditions (satellite angular rate and position) as well as a greater pointing accuracy.

In this paper, the problem of the reaction wheels control software inside the mission mode is considered for the CNES microsatellite DEMETER ([PMF99],[Bui03], [PF02], [PA06],[BPR10]). A linear PD type controller designed for small pointing error is replaced by an adaptive PD controller. Comparison of the static and adaptive laws show what improvements can be expected in particular with respect to self tuning in response to modifications in the plant dynamics. The questions of weather such control strategy could help to reduce the number of control modes and the complexity of switching from one to the other are discussed but largely left for future work.

The outline of the paper is as follows. First a section is devoted to the theoretical results. LMI results for stabilization with adaptive control for any 'almost stable' system is given. Section 3 exposes the Demeter satellite attitude control problem which is solved in Section 4. Finally some conclusions are driven.

\section{Notations}

$\mathrm{R}^{m \times p}$ is the set of real $m$ by $p$ matrices. $A^{T}$ is the transpose of the matrix $A . \operatorname{Tr}(A)$ is the trace of $A . \mathbf{1}$ and $\mathbf{0}$ are respectively the identity and the zero matrices of appropriate dimensions. For symmetric matrices, $A>(\geq) B$ means that $A-B$ is positive (semi) definite. $\|A\|_{\bullet}=\sqrt{\operatorname{Tr}\left(A A^{T}\right)}$ is the Frobenius norm of A.

$\phi\left(\mathrm{R}^{m \times p} \rightarrow \mathrm{R}^{m \times p}\right)$ is a function defined by

$$
\phi(K)=\psi\left(\|K\|_{\bullet}^{2}\right) \cdot K
$$

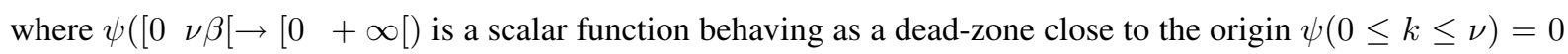
and $\psi(\nu \leq k<\nu \beta)$ and a monotone increasing barrier function that goes to infinity as $k$ converges to $\nu \beta$. An example of such function is $\psi(\nu \leq k<\nu \beta)=\frac{k-\nu}{\nu \beta-k}$. 


\section{Main results}

Linear MIMO systems described in state-space are considered

$$
\dot{x}=A x+B u, y=C x .
$$

where $x \in \mathrm{R}^{n}$ is the state, $u \in \mathrm{R}^{m}$ is the control input and $y \in \mathrm{R}^{p}$ are the measured outputs. It is assumed that $p \geq m$, i.e. there are more outputs than inputs.

It is assumed that a static output-feedback $u=F_{0} y$ has been designed such that the closed-loop system $\dot{x}=A\left(F_{0}\right) x$ is asymptotically stable where

$$
A(F)=A+B F C .
$$

No assumption is made about the closed-loop passivity but the following proposition gives a way to define passive outputs for that closed-loop system.

Proposition 1 If $\dot{x}=A\left(F_{0}\right) x$ is asymptotically stable then there exists $(P, G, \mu)$ solution to the following LMI problem

$$
P>\mathbf{0},\left[\begin{array}{cc}
A^{T}\left(F_{0}\right) P+P A\left(F_{0}\right) & P B-C^{T} G^{T} \\
B^{T} P-G C & -\mu \mathbf{1}
\end{array}\right]<\mathbf{0} .
$$

The pair $(G, \mu)$ is then such that the following system is passive

$$
\dot{x}=A\left(F_{0}\right) x+B w, \quad z=G C x+\frac{1}{2} \mu w .
$$

Proof Let $x^{T} P x, P>\mathbf{0}$, be a quadratic Lyapunov function proving asymptotic stability of $\dot{x}=A\left(F_{0}\right) x$, it is such that there exists $\epsilon>0$ such that $A^{T}\left(F_{0}\right) P+P A\left(F_{0}\right) \leq-\epsilon \mathbf{1}$. Then take any matrix $G$ and a large enough scalar $\mu$ such that

$$
\left(P B-C^{T} G^{T}\right)\left(\mu^{-1} \mathbf{1}\right)\left(B^{T} P-G C\right) \leq \epsilon \mathbf{1} .
$$

A Schur complement argument gives the LMI in (2). Passivity of the closed-loop system is direct, based, for example, on results in [BGFB94].

Having obtained some passivity property of the system, the following theorem allows to prove closed-loop stability with simple adaptive control.

Theorem 1 Let $\beta>1$ be a given scalar. If $\left(F_{0}, G, \mu\right)$ are feasible values for LMIs (2), then there exists $(Q, R, T, F, \alpha)$ solutions to the following LMI problem

$$
\begin{gathered}
{\left[\begin{array}{cc}
R & Q B-C^{T} G^{T} \\
B^{T} Q-G C & \mu \mathbf{1}
\end{array}\right] \geq \mathbf{0},} \\
{\left[\begin{array}{cc}
T & \left(F-F_{0}\right)^{T} \\
\left(F-F_{0}\right) & \mu^{-1} \mathbf{1}
\end{array}\right] \geq \mathbf{0}, \operatorname{Tr}(T) \leq \alpha,} \\
Q>\mathbf{0}, \begin{array}{l}
A^{T}\left(F_{0}\right) Q+Q A\left(F_{0}\right)+\alpha \beta C^{T} C \\
+R+C^{T}\left(G^{T}\left(F-F_{0}\right)+\left(F-F_{0}\right)^{T} G\right) C<\mathbf{0} .
\end{array}
\end{gathered}
$$

The solution is such that $u=F y$ is a stabilizing static output feedback satisfying $\left\|F-F_{0}\right\|_{\bullet}^{2} \leq \nu=\alpha \mu^{-1}$ and, whatever $\Gamma>\mathbf{0}$, the values $\left(\Gamma, G, \nu=\alpha \mu^{-1}, \beta\right)$ define a stabilizing adaptive controller:

$$
\begin{aligned}
& u(t)=K(t) y(t), \\
& \dot{K}(t)=-G y(t) y^{T}(t) \Gamma-\phi\left(K(t)-F_{0}\right) \Gamma .
\end{aligned}
$$

Before providing the proof we recall two technical lemmas slightly modified from [PF08]. Proofs are very similar to those in [PF08] and therefore not reproduced here. 
Lemma 1 If $y(t)$ is bounded for all $t \geq 0$, then $K(t)$ constrained by (7) is bounded such that

$$
\left\|K(t)-F_{0}\right\|_{\bullet}^{2}<\nu \beta, \forall t \geq 0 .
$$

Lemma 2 For all $(F, K)$ satisfying

$$
\left\|F-F_{0}\right\|_{\bullet}^{2} \leq \nu, \quad\left\|K-F_{0}\right\|_{\bullet}^{2}<\nu \beta
$$

the inequality $\operatorname{Tr}\left(\phi\left(K-F_{0}\right)(K-F)^{T}\right) \geq \mathbf{0}$ holds.

Proof of Theorem 1

Firstly let us prove the existence of a solution to the LMIs. Assume $\left(P, F_{0}, G, \mu\right)$ satisfy the LMIs (2). A Shur complement argument implies that

$$
A^{T}\left(F_{0}\right) P+P A\left(F_{0}\right)+\left(P B-C^{T} G^{T}\right)\left(\mu^{-1} \mathbf{1}\right)\left(B^{T} P-G C\right)<\mathbf{0}
$$

Take any small enough $\epsilon$ such that

$$
\begin{gathered}
A^{T}\left(F_{0}\right) P+P A\left(F_{0}\right)+R<\mathbf{0}, \\
R=\left(P B-C^{T} G^{T}\right)\left(\mu^{-1} \mathbf{1}\right)\left(B^{T} P-G C\right)+\epsilon \mathbf{1} .
\end{gathered}
$$

A converse Shur complement argument implies (4) and (6) with $F=F_{0}$ and $\alpha=0$. For that choice of $F=F_{0}$ (5) trivially holds with $T=\mathbf{0}$. Small perturbation argument implies the existence of a solution with (possibly small) non zero $\alpha$.

Now, let us prove the properties of $F$. Pre and post multiply (4) by $\left[\begin{array}{lll}1 & -C^{T}\left(F-F_{0}\right)^{T}\end{array}\right]$ and its transpose respectively to get

$$
\begin{aligned}
& Q B\left(F-F_{0}\right) C+C^{T}\left(F-F_{0}\right)^{T} B^{T} Q \\
& -\mu C^{T}\left(F-F_{0}\right)^{T}\left(F-F_{0}\right) C \\
& \leq R+C^{T}\left(G^{T}\left(F-F_{0}\right)+\left(F-F_{0}\right)^{T} G\right) C .
\end{aligned}
$$

Combined to (6) it gives

$$
A^{T}(F) Q+Q A(F)+C^{T}\left(\alpha \beta \mathbf{1}-\mu\left(F-F_{0}\right)^{T}\left(F-F_{0}\right)\right) C<\mathbf{0} .
$$

A Schur complement argument on (5) gives

$$
\alpha \geq \operatorname{Tr}(T) \geq \mu\left\|F-F_{0}\right\|_{\bullet}^{2}
$$

which is the expected bound on $F-F_{0}$. Reminding that $\beta>1$ and $\left(F-F_{0}\right)^{T}\left(F-F_{0}\right) \leq\left\|F-F_{0}\right\|_{\bullet}^{2} \mathbf{1}$, it also implies stability of the closed-loop: $A^{T}(F) Q+Q A(F)<\mathbf{0}$.

We now prove the stability of the closed-loop with the adaptive control law with the following Lyapunov function:

$$
V(x, K)=x^{T} Q x+\operatorname{Tr}\left((K-F) \Gamma^{-1}(K-F)^{T}\right)
$$

which derivative along the trajectories of the closed-loop system writes

$$
\dot{V}=2 x^{T} Q(A x+B K y)+2 \operatorname{Tr}\left(\dot{K} \Gamma^{-1}(K-F)^{T}\right) .
$$

Pre and post multiply (6) by $x^{T}$ and $x$ respectively to get

$$
2 x^{T} Q\left(A x+B F_{0} y\right) \leq-\alpha \beta y^{T} y-x^{T} R x-2 y^{T}\left(F-F_{0}\right)^{T} G y
$$

Thus one gets

$$
\begin{aligned}
\dot{V} \leq & 2 x^{T} Q B\left(K-F_{0}\right) y-\alpha \beta y^{T} y \\
& -x^{T} R x-2 y^{T}\left(F-F_{0}\right)^{T} G y+2 \operatorname{Tr}\left(\dot{K} \Gamma^{-1}(K-F)^{T}\right) .
\end{aligned}
$$




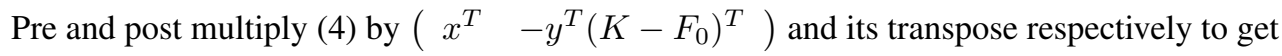

$$
\begin{array}{r}
2 x^{T} Q B\left(K-F_{0}\right)^{T} y-x^{T} R x-2 y^{T}\left(K-F_{0}\right)^{T} G y \\
\leq \mu y^{T}\left(K-F_{0}\right)^{T}\left(K-F_{0}\right) y
\end{array}
$$

and therefore

$$
\begin{aligned}
\dot{V} \leq & y^{T}\left(\mu\left(K-F_{0}\right)^{T}\left(K-F_{0}\right)-\alpha \beta \mathbf{1}\right) y \\
& +2 y^{T}(K-F)^{T} G y+2 \operatorname{Tr}\left(\dot{K} \Gamma^{-1}(K-F)^{T}\right) .
\end{aligned}
$$

Thanks to Lemma 1 , the first term is known to be negative. Noting that

$$
y^{T}(K-F)^{T} G y=\operatorname{Tr}\left(G y y^{T}(K-F)^{T}\right)
$$

and replacing $\dot{K}$ by its value one gets

$$
\dot{V} \leq-2 \operatorname{Tr}\left(\phi\left(K-F_{0}\right)(K-F)^{T}\right)
$$

and concludes the proof thanks to Lemma 2.

At this stage the results can seem confusing. Theorem 1 gives, at the knowledge of a stabilizing SOF gain $F_{0}$, two alternative controllers. One is another SOF gain $F$. The other is a 'simple' adaptive control gain, still more complex than the SOF control laws. The justification of $F$ is to be an intermediate variable used to define the Lyapunov function for the adaptive law. What is expected is that the adaptive law will have better robustness properties than the original SOF $u=F_{0} y$.

The adaptive control law is such that the gain $K$ varies around the original stabilizing gain $F_{0}$. It cannot escape from the bounded set $\left\|K-F_{0}\right\|_{\bullet}^{2} \leq \nu \beta$ (see Lemma 1) and as the system stabilizes it converges to the bounded set $\left\|K-F_{0}\right\|_{\bullet}^{2} \leq \nu$. Trivially one cannot expect any improvement from the adaptive control if $\nu$ is small. One should therefore seek for solutions maximizing $\nu$. To this end, the following design procedure is proposed:

1- Minimize $\mu$ subject to (2) with the additional constraints

$$
P>\mathbf{1}, \quad\left[\begin{array}{cc}
g^{2} \mathbf{1} & G \\
G^{T} & 1
\end{array}\right]>\mathbf{0}, \quad \mu_{0} \leq \mu
$$

where $g$ is some upper-bound on the norm of $G$ and $\mu_{0}$ is a lower-bound on $\mu_{0}$. Both these parameters should be chosen a priori based on practical considerations: $g$ large enough in order to have a feasible solution, $\mu_{0}$ small but large enough to make $\mu$ invertible in practice in the next step.

2- For the obtained $(G, \mu)$ maximize $\alpha$ subject to $(4,5,6)$.

The important feature of the design procedure is that it is guaranteed to succeed when starting from a stabilizing static output feedback $F_{0}$. The only assumption on the system is that it should be 'almost stable' thus relaxing usual 'almost passive' assumptions of [KBS94] (existence of a static output feedback that makes the system stable and passive).

A second feature is the boundedness of the control gain $K(t)$. While the usual passivity conditions conclude with the possibility of the control gain $K(t)$ to diverge in the direction $K(t)=-k(t) G$ with a positive possibly large scalar $k(t)$, our new result based on the introduction of a fictive feedthrough term $\frac{1}{2} \mu$ forbids such high gain control. The corrective term $\phi$ is intended to prevent any divergence. It not only keeps $K(t)$ is a domain of implementable values but plays a key role in the proof of stability.

The two step design procedure amounts to solving two LMIs. Solving these can be done in polynomial time using efficient semi-definite programming solvers such as SeDuMi by [Stu99]. Results of the next sections are obtained using this solver and are coded in Matlab(c) with the help of YALMIP interface of [Löf04]. Computation time for the small size problems we have considered are of less than one second.

The fact that results are formulated in terms of LMIs gives also the opportunity to extend them to guarantee robustness. Inspired by first results of [PFA08, BYYS07] extensions of this paper contributions to robustness issues are given in [PAMF11].

The remaining of the present paper is now devoted to illustrating the results on an application example. 


\section{Demeter satellite benchmark}

The AOCS control loop of DEMETER is fully described in [PA06]. In this section, only the main elements used for the study are recalled.

The AOCS reaction wheel control loop is presented at Figure 1.

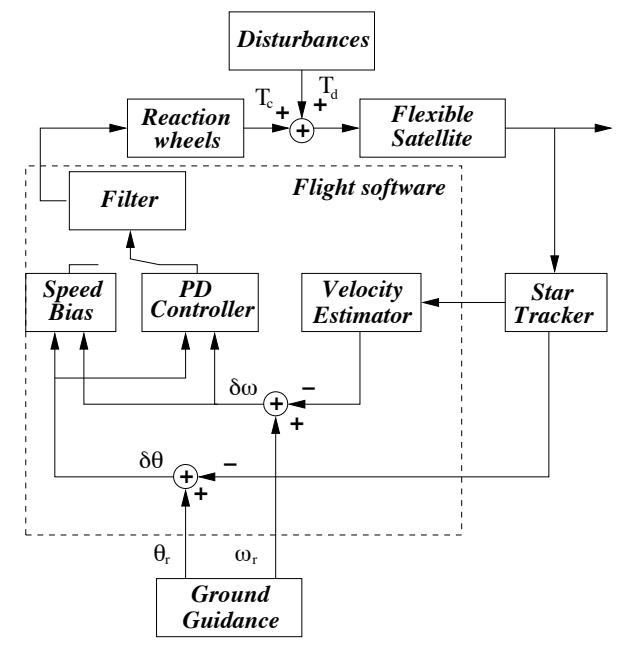

Figure 1: DEMETER MNO control loop

\subsection{Flexible satellite}

The three axes of the satellite are assumed independent. Each one is modeled as a double integrator scaled by inertia:

$$
J_{i} \dot{\omega}_{i}=T_{i}, \quad \dot{\theta}_{i}=\omega_{i}
$$

where $T$ is the total torque (actuators and disturbances) applied to the satellite to which we add two pairs of poles and zeros representing the first flexible modes. For the second axis this fourth order model (obtained by reduction of a eleventh order model containing four flexible modes) is

$$
G_{\mathrm{sat}, 2}(s)=\frac{0.04736 s^{2}+0.0006546 s+0.2991}{s^{4}+0.01387 s^{3}+6.338 s^{2}} .
$$

We shall design the adaptive control for this axis and then test it on the first axis for which the model is

$$
G_{\mathrm{sat}, 1}(s)=\frac{0.03933 s^{2}+0.0005437 s+0.2485}{s^{4}+0.01706 s^{3}+7.797 s^{2}} .
$$

For simplicity reasons, the attitude measurement is considered perfect (no star tracker noise or delay nor availability problem) and the guidance reference is zero for attitude and rate. The reaction wheel is also considered perfect (unitary gain transfer function).

\subsection{Flight software}

The flight software for the wheel control is composed of the attitude and rate estimation and the reaction wheel control law.

The attitude estimate is considered equal to the attitude measurement. The satellite angular velocity is derived from the attitude estimate via high pass filters of first order, with a time constant of 0.5 seconds. $G_{\text {estim }}(s)=\frac{s}{s+0.5}$. 
The reaction wheels control filters consists of three parts: a nonlinear control law for large pointing error switching with a PD controller for small pointing errors, both followed by the same stabilising linear filter.

The large pointing error control is intended to decrease the pointing error, with minimal reaction wheel effort. It is achieved through a nonlinear control law with the following type

$$
T_{i}=-k_{i}\left(\delta \omega_{i}+b_{i} \operatorname{sign}\left(\delta \theta_{i}\right)\right)
$$

where $T_{i}$ is the computed torque on axis $i$. At this stage of the study, this mode is not studied.

The small pointing error control is applied as soon as the pointing falls within a given threshold. The law is of proportional-derivative type such as :

$$
T_{i}=-K_{p i} \delta \theta_{i}-K_{d i} \delta \omega_{i}
$$

where $K_{p i}$ and $K_{d i}$ are respectively the proportional and derivative gains on axis $i$. Based on experiments and physical knowledge the following gains have been chosen for the satellite: $K_{p i}=0.1, K_{d i}=2$.

But for these values the closed-loop does not perform as required and a stabilizing filter is added. The synthesis of the stabilizing filter is not detailed here. Some information about the synthesis can be found in [PMF99]. The 3 SISO control filters are the result of a multi-objective $H_{2} / H_{\infty}$ LMI synthesis with pole placement in an LMI region. They have been open-loop reduced, digitalised using a first order hold method, decomposed into the summation of a static gain and two second order strictly proper filters and finally the coefficients truncated to five digits. The result consists in a PID with a low pass filter. For the second axis the filter gain is

$$
G_{\text {filter }, 2}(s)=\frac{0.5411 s^{4}-3.678 s^{3}-4.99 s^{2}-1.747 s-0.1241}{0.25 s^{6}+1.961 s^{5}+5.094 s^{4}+5.722 s^{3}+3.068 s^{2}+0.5784 s}
$$

\section{Adaptive control of the Demeter satellite}

In order to evaluate the adaptive control on this benchmark we have considered at this stage of our study the situation when the estimator and the stabilizing filter are given and we aim at replacing the static PD gain in (10) by the adaptive law (7). It amounts to applying the exposed procedure to the following system with one input and two outputs

$$
\left[\begin{array}{c}
1 \\
G_{\text {estim }}(s)
\end{array}\right] G_{\text {sat }, 2}(s) G_{\text {filter }, 2}(s) .
$$

The advantage of the considered problem is that it gives the possibility to plot in $2 \mathrm{D}$ the time histories of the gains and thus illustrate the adaptive law properties.

As a start we have applied the adaptive control design procedure starting with the known stabilizing gain

$$
F_{0}=\left[\begin{array}{ll}
K_{p 2} & K_{d 2}
\end{array}\right]=\left[\begin{array}{ll}
0.1 & 2
\end{array}\right] .
$$

Comparison of the static feedback (using gain $F_{0}$ ) with the adaptive law in terms of output signals is given in Figure 2. Simulations are done with non zero initial conditions (randomly chosen). The dotted line correspond to the responses for the static control and the solid line for the adaptive control.

The adaptive law gives a slightly faster convergence of the outputs. But the improvement is not significant. This is not surprising since the filters have been designed to optimally solve the problem with this choice of static PD control. We therefore have considered an altered case for which the improvements due to the adaptive control are more significant. To do so we have changed the static gains into

$$
F_{0}=\left[\begin{array}{ll}
K_{p 2} & K_{d 2}
\end{array}\right]=\left[\begin{array}{ll}
0.3 & 2
\end{array}\right]
$$

and performed the whole design again. It is now described step by step in details.

At the first step of the procedure we chose $g=10^{3}$ and $\mu_{0}=10^{-4}$ and found the following values

$$
G=\left[\begin{array}{ll}
28.131 & -165.556
\end{array}\right], \mu=196.494 .
$$



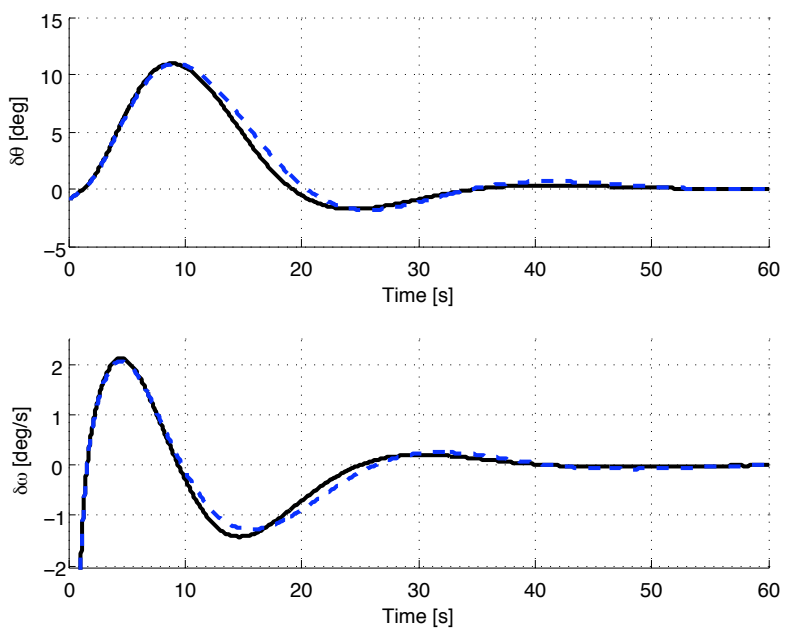

Figure 2: $y(t)$ with static and adaptive control for $F_{0}=\left[\begin{array}{ll}0.1 & 2\end{array}\right]$

For the second step of the procedure we chose $\beta=2$ and found

$$
F=\left[\begin{array}{ll}
0.1437 & 2.0000
\end{array}\right], \alpha=4.7993 .
$$

To apply the adaptive control it is then needed to chose $\Gamma$ and $\phi$. For the choice of $\Gamma$ we proceed as follows. We assume that the PD control dedicated to small pointing error situations intended to work for values of $y$ close to zero, or at least for $\|y\| \leq y_{\max }=20$. The adaptation gain is subject at first approximation to $\dot{K}=-G y y^{T} \Gamma$. Its derivative should not be too large to be implementable. From practical consideration we decided that a limitation $\|\dot{K}\|<\dot{K}_{\max }=1$ would be reasonable. This meant in first approximation to take $\Gamma<\dot{K}_{\max } / y_{\max }^{2}$ and we took $\Gamma=1.489 \cdot 10^{-5}$.

For the choice of $\phi$ we proceeded to some tests and ended in choosing

$$
\phi(\nu \leq k \leq \nu \beta)=\left(10^{3}(k-\nu)+\frac{k-\nu}{\nu \beta-k}\right)^{4}
$$

which does satisfy the assumptions and makes it a rapidly growing barrier as $k$ gets out of the region bounded by $\nu$.

The time histories of the outputs $y(t)=\left(\begin{array}{lll}\delta \theta(t) & \delta \omega(t)\end{array}\right)^{T}$ (Figure 3), the control signals $u(t)=K(t) y(t)$ (Figure 4) and the control gains $K(t)$ (Figure 5) are all compared to the static control $F_{0}=\left[\begin{array}{ll}0.3 & 2\end{array}\right]$ (doted line). The plots clearly show the improvement due to the adaptation which is not at the expense of highly varying gains or larger control inputs.

In order to see the influence of the function $\phi$ we also give in Figure 6 the time histories of $K(t)$ with $K_{p}$ on the horizontal axis and $K_{d}$ on the vertical axis. The inner dotted circle corresponds to $\left\|K-F_{0}\right\|_{\bullet}=\nu$ and the outer circle is $\left\|K-F_{0}\right\|_{\bullet}=\nu \beta$. The expected bounds on the control gains are indeed satisfied and would be maintained even in case of bounded noise in the loop, including noise on the measurements.

To test the robustness of the adaptive control it is now applied to the model of the first axis of the satellite:

$$
\left[\begin{array}{c}
1 \\
G_{\mathrm{estim}}(s)
\end{array}\right] G_{\mathrm{sat}, 1}(s) G_{\mathrm{filter}, 2}(s) .
$$

We intentionally kept the same filter $G_{\text {filter,2 }}(s)$ which is not designed for this axis. The result is that the closedloop contains a stable but very slow oscillating mode which cannot be damped with PD control. This oscillation can thus be seen as some perturbation. Figure 7 shows the time histories of the outputs in that case. It can be clearly seen that the adaptive control is most effective. The time histories of the gains are quite similar to those in Figures 5 and are therefore not reproduced. 

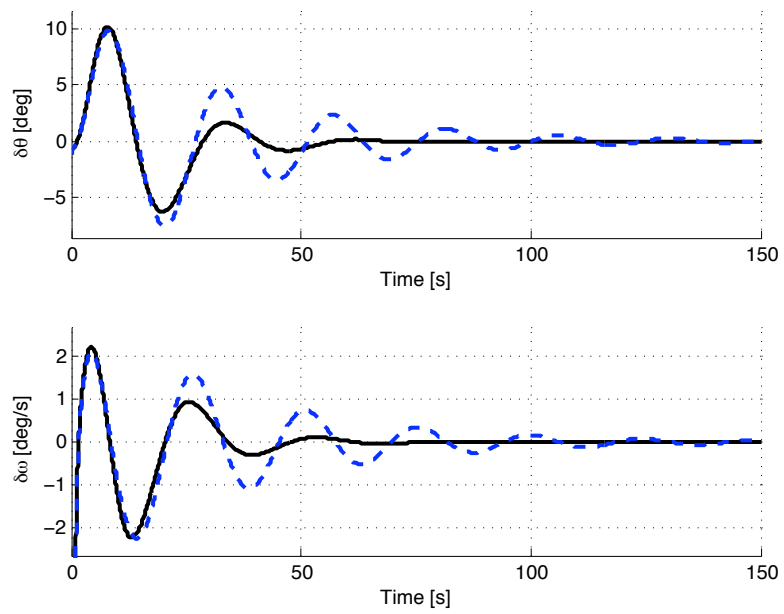

Figure 3: $y(t)$ with static and adaptive control for $F_{0}=\left[\begin{array}{ll}0.3 & 2\end{array}\right]$

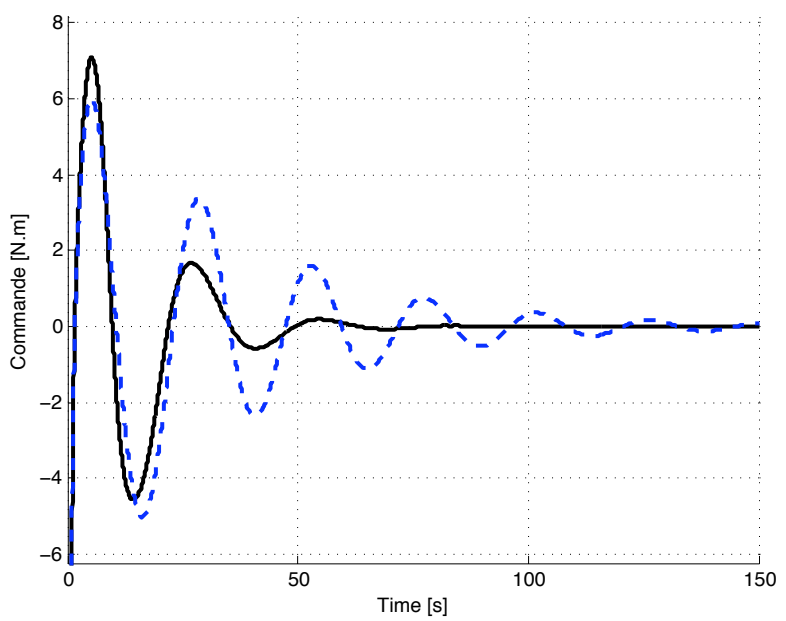

Figure 4: $u(t)$ with static and adaptive control for $F_{0}=\left[\begin{array}{ll}0.3 & 2\end{array}\right]$

The simulations show that the simple adaptive control can be of interest for improving a given linear control law. Here we have tested the adaptation of the PD gains and further experiments will be done for adaptation of the filter gains.

Another prospective work will be devoted to the originally formulated problem of reducing the number of control 'modes'. Currently the satellite is controlled with the two modes defined by (9) in case of large pointing errors and the PD control (10) for small pointing error. Keeping the properties of these two modes, an alternative, unique mode control would be of the type

$$
T_{i}=-K_{p i} \mathrm{sat}_{b_{i}}\left(\delta \theta_{i}\right)-K_{d i} \delta \omega_{i}
$$

where $\mathrm{sat}_{b_{i}}$ stands for the saturation operator with lower-bound $-b_{i}$ and upper bound $b_{i}$. This saturated representation of the switched static control has been studied in [BPR10]. In cooperation with the authors of this paper we will consider the adaption of the gains of that saturated PD control and to proving global stability of the non-linear closed loop. 

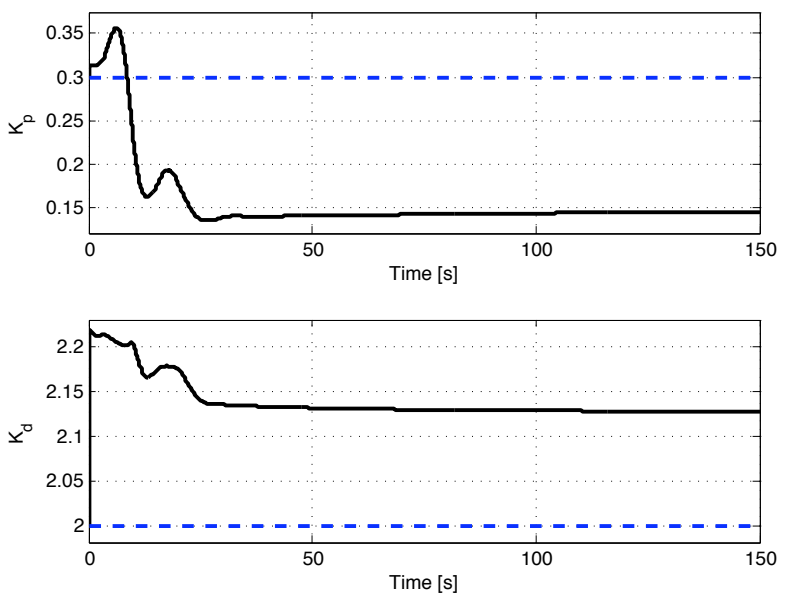

Figure 5: $K(t)$ with static and adaptive control for $F_{0}=\left[\begin{array}{ll}0.3 & 2\end{array}\right]$

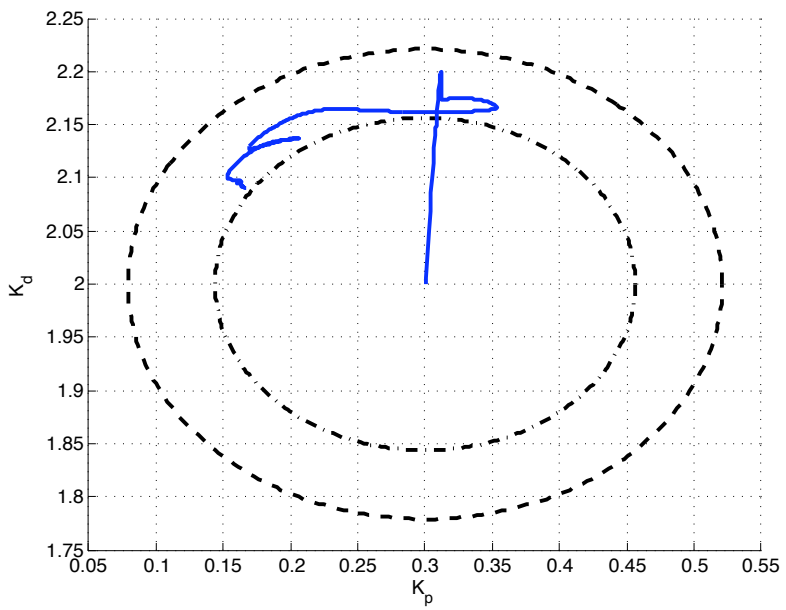

Figure 6: $K(t)$ with static and adaptive control for $F_{0}=\left[\begin{array}{ll}0.3 & 2\end{array}\right]$

\section{Conclusions}

The paper produces a new theoretical result for direct adaptive control. Namely it relaxes 'almost passive' conditions into 'almost stable' conditions. The result is that any given LTI control law can be converted into an adaptive version of it with gains kept close to the original ones while preserving stability properties. The benefit of such adaptation is possibly improved convergence and robustness. This is illustrated on a real application satellite model. Only two scalar gains of an LTI controller are adapted in the simulations, future work will be devoted to testing adaptation on other gains of the control law.

\section{References}

[ÅW89] K.J. Åström and B. Wittenmark. Adaptive Control. Addison-Wesley, 1989.

[BGFB94] S. Boyd, L. El Ghaoui, E. Feron, and V. Balakrishnan. Linear Matrix Inequalities in System and Control Theory. SIAM Studies in Applied Mathematics, Philadelphia, 1994. 

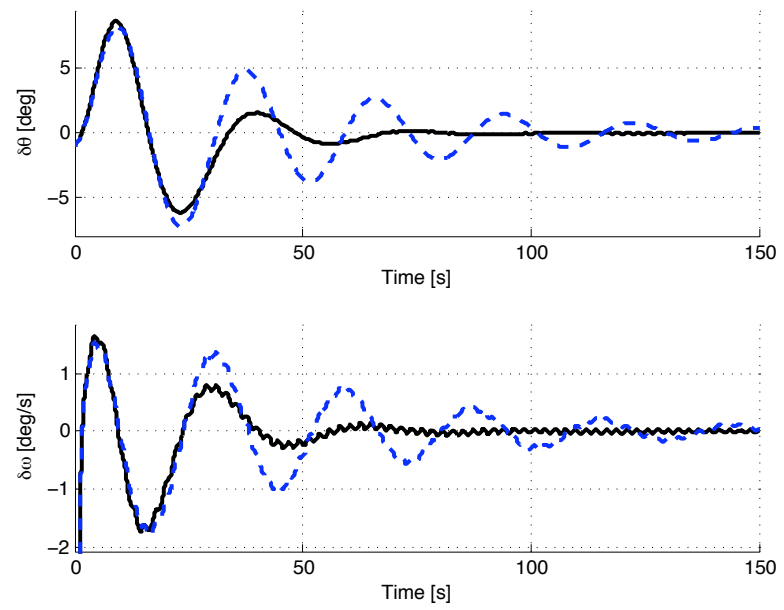

Figure 7: $y(t)$ for the controls applied to the first axis

[BPR10] J.-M. Biannic, C. Pittet, and C. Roos. Lpv analysis of switched controllers in satellite attitude control systems. In AIAA Guidance, Navigation, and Control Conference, Toronto, August 2010.

[BTH06] I. Barkana, M.C.M. Teixeira, and L. Hsu. Mitigation of symmetry condition in positive realness for adaptive control. Automatica, 42(9):1611-1616, September 2006.

[Bui03] F. Buisson. The DEMETER program: a pathfinder to a high performance microsatellite line. In AIAA USU conference on small satellites, pages 441-446, Logan, Utah, USA, 2003.

[BYYS07] R. Ben Yamin, I. Yaesh, and U. Shaked. Simplified adaptive control with guaranteed $H_{\infty}$ performance. In IFAC Workshop on Adaptation and Learning in Control and Signal Processing, St. Petersburg, August 2007.

[Fra74] A.L. Fradkov. Adaptive stabilization of a linear dynamic plant. Autom. Remote Contr., 35(12):19601966, 1974.

[Fra03] A.L. Fradkov. Passification of non-square linear systems and feedback Yakubovich-Kalman-Popov lemma. European J. of Control, 6:573-582, 2003.

[Fra08] A.L. Fradkov. Passification of linear systems with respect to given output. In IEEE Conf. Decision and Control, pages 646-651, 2008.

[IS96] P. Ioannou and J. Sun. Robust Adaptive Control. Prentice Hall, Inc, 1996.

[KBS94] H. Kaufman, I. Barkana, and K. Sobel. Direct adaptive control algorithms. Springer, New York, 1994.

[Löf04] J. Löfberg. YALMIP : A Toolbox for Modeling and Optimization in MATLAB, 2004.

[PA06] C. Pittet and D. Arzelier. DEMETER: a benchmark for robust analysis and control of the attitude of flexible microsatellites. In IFAC Symposium on Robust Control Design, Toulouse, France, 2006.

[PAMF11] D. Peaucelle, B. Andrievsky, V. Mahout, and A. Fradkov. Robust simple adaptive control with relaxed passivity and PID control of a helicopter benchmark. In IFAC World Congress, 2011.

[PF02] C. Pittet and C. Fallet. Gyroless attitude control of a flexible microsatellite. In Dynamics and control of systems and structures in space, pages 65-71, Cambridge, UK, 2002.

[PF08] D. Peaucelle and A.L. Fradkov. Robust adaptive $L_{2}$-gain control of polytopic MIMO LTI systems LMI results. Systems \& Control Letters, 57(11):881-887, 2008. 
[PFA08] D. Peaucelle, A. Fradkov, and B. Andrievsky. Passification-based adaptive control of linear systems: Robustness issues. Int. J. of Adaptive Control and Signal Processing, 22(6):590-608, August 2008. doi: $10.1002 /$ acs.1009.

[PMF99] C. Pittet, J. Mignot, and C. Fallet. LMI based multi-objective $H_{\infty}$ control of flexible microsatellites. In IEEE Conference on Decision and Control, Sydney, Australia, 1999.

[Stu99] J.F. Sturm. Using SeDuMi 1.02, a MATLAB toolbox for optimization over symmetric cones. Optimization Methods and Software, 11-12:625-653, 1999. 